\title{
B 样条在一些渐近组合问题中的应用
}

\author{
许艳*，王仁宏
}

大连理工大学数学科学学院, 大连 116024

E-mail: yanxudlut@yahoo.cn, renhong@dlut.edu.cn

收稿日期: 2010-03-12; 接受日期: 2010-07-07; * 通信作者

国家自然科学基金 (批准号: 60373093, 60533060, 10801024, 10726068, 11071031)、NSFC- 广东联合基金 (批准号: U0935004) 和 中央高校基本科研业务费专项资金资助项目

摘要 本文考察了 $\mathrm{B}$ 样条函数及其导数的渐近性质, 并给出了收玫阶; 考察了经典 Eulerian 数和两类 广义 Eulerian 数的渐近性质; 给出了以 Hermite 多项式表示的细化 Eulerian 数的渐近形式. Carlitz 等 人利用中心极限定理得到 Eulerian 数渐近公式的逼近阶为 $\frac{3}{4}$ 阶. 利用样条方法, 我们得到更为精确的 逼近阶. 将样条方法引入到组合数的渐近分析中, 为离散对象的研究提供了一种新的分析方法.

关键词 B 样条 Eulerian 数 细化 Eulerian 数 下降多项式 渐近逼近

MSC (2000) 主题分类 $05 \mathrm{~A} 16,41 \mathrm{~A} 15$

\section{1 引言}

众所周知, 产生于逼近论的样条函数在计算几何及小波等领域中有着较为重要的应用. 但是由于 样条理论与研究离散对象的组合数学两个学科之间相去较远, 因而样条理论较少应用于组合数学问题 的研究中. 本文旨在探索样条理论在渐近组合学中的应用.

Eulerian 多项式自从 1775 年由 Euler 在其著作 “Institutiones calculi differentialis cum eius usu in analysi finitorum ac Doctrina serierum” (Chapter VII) 中介绍以来, 已经被深入的研究. 一般而 言, Eulerian 数有三种解释: 组合解释、概率解释和几何解释. 这三种解释业已发展成为成熟的研究 Eulerian 多项式的三种方法. 本文的主要目的是用样条视角考察 Eulerian 数, 给出经典 Eulerian 数和 两类广义 Eulerian 数的样条解释. 依照这种观点, 我们可以利用样条函数的渐近性质得到一系列组合 数的渐近形式.

B 样条渐近性质的研究, 最早可以追溯到 1904 年. Sommerfeld ${ }^{[1]}$ 从力学问题出发, 证明了 B 样 条随着次数的增大, 渐近到 Gauss 函数. 此后 Schoenberg 等 ${ }^{[2]}$ 证明了一般结点的 B 样条随着次数 的增大, 渐近到 Pólya 频率函数. 1992 年, Unser 和他的合作者 ${ }^{[3]}$ 证明了基数 B 样条的渐近性质在 $L^{p}(\mathbb{R})$ 空间中仍然成立. Brinks ${ }^{[4]}$ 将 Unser 的结果推广到了 B 样条的导数情形, 并应用于构造新的小 波基底. 但是他们都没有对逼近阶进行讨论. 在此, 我们重新讨论了 $\mathrm{B}$ 样条及其导数在 $L^{p}$ 空间中的 收玫性, 并给出了逼近阶; 利用该结果给出了经典 Eulerian 数和两类广义 Eulerian 数的渐近性质; 从 而为渐近组合学的研究提供了一种新的分析方法.

本文分为以下几个部分. 第 2 节讨论了 $\mathrm{B}$ 样条及其导数在 $L^{p}$ 空间中的渐近性质, 并给出了收敛 阶. 第 3 节利用 Eulerian 数、细化 Eulerian 数和下降多项式的几何解释, 给出了三类组合数的样条解 释. 第 4 节利用 B 样条的渐近性质和三类组合数的样条解释导出这三类组合数的渐近性质. 


\section{B 样条的渐近性质}

$d$ 阶 $\mathrm{B}$ 样条记作 $B_{d}$, 定义为

$$
B_{1}(x)= \begin{cases}1, & \text { 若 } x \in[0,1), \\ 0, & \text { 否则 },\end{cases}
$$

当 $d \geqslant 2$ 时,

$$
B_{d+1}(x)=\frac{x}{d} B_{d}(x)+\frac{d+1-x}{d} B_{d}(x-1)
$$

且有如下显式表达式

$$
B_{d}(x)=\frac{1}{(d-1) !} \sum_{i=0}^{d}\left(\begin{array}{l}
d \\
i
\end{array}\right)(-1)^{i}(x-i)_{+}^{d-1},
$$

其中 $(x-i)_{+}^{d-1}:=(\max \{x-i, 0\})^{d-1}$.

若 $f \in L^{1}(\mathbb{R})$ 或 $L^{2}(\mathbb{R})$, 则 Fourier 变换 $f^{\wedge}$ 和 Fourier 逆变换 $f^{\vee}$ 定义为

$$
\begin{aligned}
& f^{\wedge}(\omega):=\int_{-\infty}^{+\infty} f(t) e^{-i \omega t} d t, \\
& f^{\vee}(\omega):=\frac{1}{2 \pi} \int_{-\infty}^{+\infty} f(t) e^{i \omega t} d t .
\end{aligned}
$$

将基数 B 样条做一个平移 $M_{d}(x)=B_{d}\left(x+\frac{d}{2}\right)$, 得到关于原点对称的中心 $\mathrm{B}$ 样条 $M_{d}(x)$. 因此 $M_{d} \in$ $L^{1}(\mathbb{R}) \cap L^{2}(\mathbb{R})$, 且有如下 Fourier 变换,

$$
M_{d}^{\wedge}(\omega)=\operatorname{sinc}^{d}\left(\frac{\omega}{2}\right)
$$

其中 $\operatorname{sinc}(t)$ 为

$$
\operatorname{sinc}(t):= \begin{cases}\frac{\sin t}{t}, & t \neq 0, \\ 1, & t=0 .\end{cases}
$$

为了得到 $\mathrm{B}$ 样条及其导数的渐近性质, 首先给出以下关于 sinc 函数上界的引理.

引理 $2.1^{[4]}$ 若 $k, d \in \mathbb{N}$, 且 $d \geqslant k+2$, 存在 $c_{k} \in \mathbb{R}^{+}$使得

$$
G_{k}(x):=\chi_{\mathbb{R} \backslash[-1,1]}(x) \frac{c_{k}}{\pi^{2} x^{2}}+\pi^{k}|x|^{k} \exp \left(-x^{2}\right),
$$

其中 $\chi_{A}(x)$ 是定义在集合 $A$ 上的特征函数, 则

$$
G_{k} \in L^{p}(\mathbb{R}), \quad \forall p \in[1, \infty),
$$

且

$$
\pi^{k}|x|^{k} \cdot\left|\operatorname{sinc}\left(\frac{\pi x}{\sqrt{d}}\right)\right|^{d} \leqslant G_{k}(x) .
$$

证明 先证明如下的不等式

$$
\operatorname{sinc}(x) \leqslant 1-x^{2}, \quad \forall x \in[0,1] .
$$


首先有

$$
\operatorname{sinc}^{n}\left(\frac{x}{\sqrt{d}}\right) \leqslant\left(1-\frac{x^{2}}{d}\right)^{d} \leqslant \exp \left(-x^{2}\right), \quad \forall x \in[0, \sqrt{d}] .
$$

由此, 定义正定函数

$$
p(x)=\exp \left(x^{2}\right)\left(1-\frac{x^{2}}{d}\right)^{d} .
$$

$p(x)$ 有如下的导数形式

$$
\frac{\partial p(x)}{\partial x}=\frac{-2 \exp \left(x^{2}\right) x^{3}\left(1-x^{2} / d\right)^{d}}{d\left(1-x^{2} / d\right)}
$$

并且当 $x \in[0, \sqrt{d}]$ 时, 该导数为负值. 因此 $p(x)$ 在该区间的最大值在 $x=0$ 处.

$$
\sup _{x \in(0, \sqrt{n})} p(x)=p(0)=1,
$$

从而证明了 (2.5) 式.

其次, 当 $x \geqslant \sqrt{d}$ 时, 令

$$
c_{k}:=\max _{n \geqslant k+2}\left(\frac{d^{\frac{k+2}{2}}}{\pi^{d-k-2}}\right) .
$$

由于数列

$$
\left(\frac{d^{\frac{k+2}{2}}}{\pi^{d-k+2}}\right)_{d \geqslant k+2}
$$

的收玫性, 必然存在这样的 $c_{k}$, 使得

$$
\pi^{k}|x|^{k} \cdot\left|\operatorname{sinc}\left(\frac{\pi x}{\sqrt{d}}\right)\right|^{d}
$$

有如下上界:

$$
\pi^{k}|x|^{k} \cdot\left|\operatorname{sinc}\left(\frac{\pi x}{\sqrt{d}}\right)\right|^{d} \leqslant \frac{c_{k}}{x^{2} \pi^{2}} .
$$

从而

$$
\left(\frac{\sqrt{d}}{x \pi}\right)^{d} \leqslant\left(\frac{\sqrt{d}}{x}\right)^{k+2} \cdot \frac{1}{\pi^{d}} \leqslant \frac{c_{k}}{x^{k+2} \pi^{k+2}} .
$$

这样利用

$$
\left|\operatorname{sinc}^{d}\left(\frac{\pi x}{\sqrt{d}}\right)\right| \leqslant\left(\frac{\sqrt{d}}{\pi x}\right)^{d},
$$

可以得到独立于 $d$ 的上界 $\frac{c_{k}}{x^{2} \pi^{2}}$.

最后, 定义独立于 $d$ 的序列 $G_{k}(x)$. 适当的组合不等式 (2.5) 和 (2.6) 的右端, 从而使 $L_{p}(-\infty,+\infty)$ 中的函数 $G_{k}(x)$ 为函数

$$
\pi^{k}|x|^{k} \cdot\left|\operatorname{sinc}\left(\frac{\pi x}{\sqrt{d}}\right)\right|^{d}
$$

的一致上界, 其中 $p \in[1,+\infty)$.

基于上述引理, 我们有如下关于 $\mathrm{B}$ 样条及其导数的渐近性质定理. 
定理 1 令 $k \in \mathbb{N}$, 则对于 $d>k+2, B$ 样条的 $k$ 阶导数构成的序列 $B_{d}^{(k)}$ 收玫于 Gauss 函数的 $k$ 阶导数, 即

$$
\left(\frac{d}{12}\right)^{\frac{k+1}{2}} B_{d}^{(k)}\left(\sqrt{\frac{d}{12}} x+\frac{d}{2}\right)=\frac{1}{\sqrt{2 \pi}} D^{k} \exp \left(-\frac{x^{2}}{2}\right)+O\left(\frac{1}{d}\right),
$$

并且

$$
\lim _{d \rightarrow \infty}\left\{\left(\frac{d}{12}\right)^{\frac{k+1}{2}} B_{d}^{(k)}\left(\sqrt{\frac{d}{12}} x+\frac{d}{2}\right)\right\}=\frac{1}{\sqrt{2 \pi}} D^{k} \exp \left(-\frac{x^{2}}{2}\right),
$$

其中极限是点态收敛或者在 $L^{p}(\mathbb{R}), p \in[2, \infty)$ 中.

证明 令

$$
L_{n}(x):=d \ln \left(\operatorname{sinc}\left(\frac{x}{2} \sqrt{\frac{12}{d}}\right)\right),
$$

由于对称性, 可以假设 $x \geqslant 0$. 利用 Taylor 定理, 对于任意的 $x \in[0,1]$ 和 $d \in \mathbb{N}$, 有

$$
\operatorname{sinc}\left(\frac{x}{2} \sqrt{\frac{12}{d}}\right)=1-\frac{x^{2}}{2} \frac{1}{d}+O\left(\frac{1}{d^{2}}\right)
$$

和

$$
\ln (1+x)=x+O\left(x^{2}\right)
$$

则对任意 $x \in[0,1]$ 和 $d \in \mathbb{N}$, 有

$$
L_{n}(x)=d \ln \left(1-\frac{x^{2}}{2} \frac{1}{d}+O\left(\frac{1}{d^{2}}\right)\right)=-\frac{x^{2}}{2}+O\left(\frac{1}{d}\right) .
$$

结合公式 (2.9) 与 (2.10), 我们有

$$
\operatorname{sinc}^{d}\left(\frac{x}{2} \sqrt{\frac{12}{d}}\right)=\exp \left(-\frac{x^{2}}{2}\right)\left(1+O\left(\frac{1}{d}\right)\right) .
$$

此外, 由于 $M_{d}^{\wedge}(\omega)=\operatorname{sinc}^{d}\left(\frac{\omega}{2}\right)$ 且 $M_{d} \in C^{d-1}(\mathbb{R})$, 从而对于 $k \leqslant d-1$, 有

$$
\left[M_{d}^{(k)}\right]^{\wedge}(\omega)=i^{k} \omega^{k} \operatorname{sinc}^{d}\left(\frac{\omega}{2}\right) .
$$

因此可以得到

$$
\begin{aligned}
\left(\frac{d}{12}\right)^{\frac{k+1}{2}}\left[B_{d}^{(k)}\left(\sqrt{\frac{d}{12}} x+\frac{d}{2}\right)\right]^{\wedge}(\omega) & =i^{k} \omega^{k} \operatorname{sinc}^{d}\left(\frac{\omega}{2} \sqrt{\frac{12}{d}}\right) \\
& =i^{k} \omega^{k} \exp \left(-\frac{\omega^{2}}{2}\right)\left(1+O\left(\frac{1}{d}\right)\right) \\
& =\left[D^{k}\left\{\frac{1}{\sqrt{2 \pi}} \exp \left(-\frac{x^{2}}{2}\right) \cdot\left(1+O\left(\frac{1}{d}\right)\right)\right\}\right]^{\wedge}(\omega) .
\end{aligned}
$$

对上式做 Fourier 逆变换, 得到

$$
\begin{aligned}
\left(\frac{d}{12}\right)^{\frac{k+1}{2}} B_{d}^{(k)}\left(\sqrt{\frac{d}{12}} x+\frac{d}{2}\right) & =D^{k}\left(\frac{1}{\sqrt{2 \pi}} \exp \left(-\frac{x^{2}}{2}\right)\right)+D^{k}\left(\frac{1}{\sqrt{2 \pi}} \exp \left(-\frac{x^{2}}{2}\right)\right) O\left(\frac{1}{d}\right) \\
& =D^{k}\left(\frac{1}{\sqrt{2 \pi}} \exp \left(-\frac{x^{2}}{2}\right)\right)+O\left(\frac{1}{d}\right) .
\end{aligned}
$$

866 
则

$$
\lim _{d \rightarrow \infty}\left\{\left(\frac{d}{12}\right)^{\frac{k+1}{2}} B_{d}^{(k)}\left(\sqrt{\frac{d}{12}} x+\frac{d}{2}\right)\right\}=\frac{1}{\sqrt{2 \pi}} D^{k} \exp \left(-\frac{x^{2}}{2}\right),
$$

其中等式 (2.12) 左边的极限由引理 2.1 得到, 并且意味着点态收玫. 显然, $B_{d}^{(k)}(\omega)$ 以引理 2.1 中所定 义的 $L_{p}$ 空间中的独立于 $d$ 的函数 $G_{k}(x)$ 为上界. 利用 Lebesgue 控制收玫定理, 得到了 $B_{d}^{(k)}(\omega)$ 在 $L_{p}, p \in[1,+\infty)$ 中收玫. Titchmarsh 不等式表明, 当 $1 \leqslant p \leqslant 2$ 且 $p^{-1}+q^{-1}=1$ 时, Fourier 变换是 $L_{p}(-\infty,+\infty)$ 到 $L_{q}(-\infty,+\infty)$ 空间中的有界线性运算. 因而, 当 $q \in[2,+\infty]$ 时, 利用 Titchmarsh 不 等式, 得到 $L_{q}$ 空间中 $B_{d}^{(k)}(x)$ 的收玫性.

当 $k=0$ 时, 定理 1 退化为

推论 2.1 对于任意 $d \in \mathbb{N}$, 基数 B 样条收玫到 Gauss 函数,

$$
\sqrt{\frac{d}{12}} B_{d}\left(\sqrt{\frac{d}{12}} x+\frac{d}{2}\right)=\frac{1}{\sqrt{2 \pi}} \exp \left(-\frac{x^{2}}{2}\right)+O\left(\frac{1}{d}\right)
$$

并且有

$$
\lim _{d \rightarrow \infty} \sqrt{\frac{d}{12}} B_{d}\left(\sqrt{\frac{d}{12}} x+\frac{d}{2}\right)=\frac{1}{\sqrt{2 \pi}} \exp \left(-\frac{x^{2}}{2}\right),
$$

其中极限为点态收敛或者在 $L^{p}(\mathbb{R}), p \in[2, \infty)$ 中.

定理 1 及其推论 2.1 刻画了 B 样条及其导数的渐近性质, 可以用于组合数渐近性质的研究. 我们 将利用它们讨论后文中所要引入的 Eulerian 数等组合数的渐近性质, 并得到一系列有趣的结果, 从而 为计数组合学的研究提供一种新的样条方法.

\section{Eulerian 数的样条解释}

Laplace $^{[5]}$ 将 Eulerian 数表示成超立方体截面体积, 从而给出了 Eulerian 数的几何解释

$$
V\left(T_{k}^{d}\right)=\frac{1}{d !} A_{d, k}
$$

其中

$$
T_{k}^{d}:=\left\{x \in[0,1]^{d} \mid k-1 \leqslant \sum_{i=1}^{d} x_{i} \leqslant k, k=0, \ldots, d\right\} .
$$

$A_{d, k}$ 也可以用来表示 $S_{d}$ 对称群中所有降序数为 $k$ 的排列总数, 即

$$
A_{d, k}=\#\left\{\pi \mid \pi:=a_{1}, a_{2}, \ldots, a_{d} \in S_{d}, \# D(\pi)=k\right\},
$$

其中 \# $A$ 表示集合 $A$ 的基数, $D(\pi):=\left\{i \mid a_{i}>a_{i-1}, 1 \leqslant i \leqslant d-1\right\}$ 为降序集合.

从这个组合解释不难看出 $A_{d, k}$ 恰好是集合 $S_{k}:=\left\{x \in I^{n} \mid x_{i}>x_{i}+1\right.$, 对于 $i$ 的 $k$ 个值 $\}$ 的体积. 一个很自然的问题, 集合 $T_{k}^{d}$ 与集合 $S_{k}$ 之间是否存在一个保测度的映射使得二者同构. 这个问题最早 由 Foata ${ }^{[6]}$ 提出, 并由 Stanley ${ }^{[7]}$ 给出了构造性的证明, 从而给出了 Eulerian 数几何解释的组合证明.

Stanley 的方法极具代表性, 至今很多与体积相关联的组合数仍是用类似的构造方法得到其几何 解释. 一个很好的例子是由 Steingrímsson ${ }^{[8]}$ 给出的关于指标序列的下降多项式系数可以看作是扩张 的超立方体截面体积. 
下降多项式, 记作 $D_{d}^{n}(t)$, 定义为

$$
D_{d}^{n}(t)=\sum_{k=0}^{d} D(d, n, k) t^{k},
$$

其中 $D(d, n, k)$ 是 $S_{d}^{n}$ 中有 $k$ 个降序的指标序列的数量. 这里 $S_{d}^{n}$ 表示所有指标序列的集合. 指标序列为 $S_{d}$ 对称群中的一般序列加上一个 0 到 $n-1$ 之间的脚标 (参照 $[8,9]$ ). 当 $n=1$ 时, $D(d, 1, k)=A_{d, k+1}$, 因而 $D(d, n, k)$ 可以看做是一种广义 Eulerian 数. 近年来 Stanley ${ }^{[7]}$, Steingrímsson ${ }^{[8,9]}$, Bagno $^{[10]}$ 等人 对其进行了深入的研究. 指标序列也通常被称作着色序列. 在文献 [8] 中, Steingrímsson 给出了指标序 列的几何解释,

$$
D(d, n, k)=V\left(X_{n, k}^{d}\right)
$$

其中

$$
X_{n, k}^{d}:=\left\{x \in n \cdot[0,1]^{d} \mid(k-1) n+1 \leqslant \sum_{i=1}^{d} x_{i} \leqslant k n+1\right\} .
$$

细化 Eulerian 数, 记作 $\mathbf{A}_{d, k, j}$, 为对称群 $S_{d}$ 中有 $k$ 个降序并且最后一个元素为 $j$ 的排列总数, 即

$$
\mathbf{A}_{d, k, j}:=\#\left\{\pi \mid \pi:=a_{1}, \ldots, a_{d} \in S_{d}, \# D(\pi)=k, a_{d}=j\right\} .
$$

作为 $A_{d, k}$ 的细化形式, $\mathbf{A}_{d, k, j}$ 可以看作是相邻两 Eulerian 数 $A_{d, k}$ 与 $A_{d, k+1}$ 之间的一个插值. 自然地, 人们关心细化 Eulerian 数是否继承了 Euleiran 数的一些性质, 例如对数凹性和渐近性质.

Ehrenborg 等 [9] 将 $\mathbf{A}_{d, k, j}$ 表示成单位立方体相邻切片 $T_{k}$ 与 $T_{k+1}$ 的混合体积, 从而得到了 $\mathbf{A}_{d, k, j}$ 关于 $j$ 的对数凹性. 由于 Eulerian 数 $A_{d, k}$ 关于 $d$ 的渐近形式为 Gauss 型, 人们猜测 $\mathbf{A}_{d, k, j}$ 也 “遗传” 了同样的性质. 然而在文献 [11] 中, Conger 利用数值实验观察到 $\mathbf{A}_{d, k, j}$ 随着 $d$ 值的增大, 其渐近形 式并非正态分布的现象. 在此, 我们给出细化 Eulerian 数的样条解释; 利用这一解释给出了由 Hermite 正交多项式表示的细化 Eulerian 数的渐近形式; 从而解释了 Conger 利用数值实验观测到的现象.

基于上述三类组合数的几何解释, 在文献 [12] 中, 我们讨论了它们与 $\mathrm{B}$ 样条之间的关系. 为了叙 述的方便, 我们用 $\left[\lambda^{j}\right] f(\lambda)$ 表示给定的关于 $\lambda$ 的级数 $f(\lambda)$ 中 $\lambda^{j}$ 项的系数.

引理 3.1 ${ }^{[12]}$ 以下等式成立:

$$
\begin{aligned}
& A_{d, k}=d ! \cdot B_{d+1}(k) ; \\
& D(d, n, k)=d ! \cdot n^{d} \cdot B_{d+1}\left(k+\frac{1}{n}\right) ; \\
& \mathbf{A}_{d+1, k, d-j+1}=d ! \cdot\left[\lambda^{j}\right]\left((\lambda+1)^{d} B_{d+1}\left(k+\frac{1}{\lambda+1}\right)\right) /\left(\begin{array}{l}
d \\
j
\end{array}\right), \quad \lambda \geqslant 0 .
\end{aligned}
$$

该引理将 Eulerian 数及其推广形式看作是离散 B 样条, 从而将两个领域建立起联系. 利用样条理 论, 可以对与 Eulerian 数相关联的渐近组合问题进行研究, 在渐近组合学中引入一套样条函数方法.

\section{Eulerian 数的渐近性质}

1973 年, Tanny ${ }^{[13]}$ 利用概率论中的中心极限定理给出了 Eulerian 数的渐近形式. 此后, Carlitz 等 [14] 利用类似的方法得到了这一渐近公式的逼近阶. 但是 Carlitz 等给出的逼近阶仅是关于 $d$ 的 $\frac{3}{4}$ 阶. 
我们利用上文给出的 Eulerian 数的样条解释 (引理 3.1), 得到了 Eulerian 数的渐近公式并且给出 了精确的逼近阶.

定理 2 对于 $x_{d}=\sqrt{\frac{d+1}{12}} x+\frac{d+1}{2}$, 有

$$
\frac{1}{d !} A_{d,\left[x_{d}\right]}=\sqrt{\frac{6}{\pi(d+1)}} \exp \left(-\frac{x^{2}}{2}\right)+O\left(d^{-\frac{3}{2}}\right) .
$$

进一步, 将上述定理推广到更一般的情形, 有

定理 3 对于 $x_{d}=\sqrt{\frac{d+1}{12}} x+\frac{d+1}{2}$, 有

$$
\frac{1}{d ! \cdot n^{d}} D\left(d, n,\left[x_{d}\right]\right)=\sqrt{\frac{6}{\pi(d+1)}} \exp \left(-\frac{\left(x+\frac{1}{n}\right)^{2}}{2}\right)+O\left(d^{-\frac{3}{2}}\right) .
$$

当 $n=1$ 时, 该定理退化为定理 2 .

证明利用

$$
D(d, n, k)=d ! \cdot n^{d} \cdot B_{d+1}\left(k+\frac{1}{n}\right)
$$

和 B 样条渐近性质 (推论 2.1), 得到

$$
\sqrt{\frac{d}{12}} B_{d}\left(\sqrt{\frac{d}{12}} x+\frac{d}{2}\right)=\frac{1}{\sqrt{2 \pi}} \exp \left(-\frac{x^{2}}{2}\right)+O\left(\frac{1}{d}\right) .
$$

相应地, 对于 $x_{d}=\sqrt{\frac{d+1}{12}} x+\frac{d+1}{2}$, 有

$$
\frac{1}{d ! \cdot n^{d}} D\left(d, n,\left[x_{d}\right]\right)=\sqrt{\frac{6}{\pi(d+1)}} \exp \left(-\frac{\left(x+\frac{1}{n}\right)^{2}}{2}\right)+O\left(d^{-\frac{3}{2}}\right) .
$$

当 $n=1$ 时, 由于 $D(d, 1, k)=A_{d, k+1}$, 上式退化成定理 2 .

$n$ 次 Hermite 多项式, 记作 $H_{n}(x)$, 为定义在 $(-\infty,+\infty)$ 上以 $e^{-\frac{x^{2}}{2}}$ 为权函数的正交多项式, 即

$$
H_{n}(x)=(-1)^{n} e^{\frac{x^{2}}{2}} \frac{d^{n}}{d x^{n}} e^{\frac{-x^{2}}{2}} .
$$

作为许多经典正交多项式的极限形式, Hermite 多项式在渐近分析中有着非常重要的地位. 利用样条 理论, 我们可以得到以 Hermite 多项式表示的细化 Eulerian 数的渐近公式.

定理 4 令 $x_{d}=\sqrt{\frac{d+1}{12}}(x-1)+\frac{d+1}{2}$ 则

$$
\mathbf{A}_{d+1,\left[x_{d}\right], d-j+1}=d ! \sqrt{\frac{6}{\pi(d+1)}} \exp \left(-\frac{x^{2}}{2}\right) \sum_{i=0}^{j} \frac{1}{\left(\begin{array}{c}
d-j+i \\
i
\end{array}\right)}\left(\frac{d+1}{12}\right)^{-\frac{i}{2}} H_{i}(x)+O\left(d^{-\frac{3}{2}}\right),
$$

其中 $H_{n}(x)$ 是如下定义的 Hermite 多项式,

$$
H_{n}(x)=(-1)^{n} e^{\frac{x^{2}}{2}} \frac{d^{n}}{d x^{n}} e^{\frac{-x^{2}}{2}} .
$$

证明 令

$$
p(\lambda)=\sum_{j=0}^{d}\left(\begin{array}{l}
d \\
j
\end{array}\right) \mathbf{A}_{d+1, k, d-j+1} \lambda^{j},
$$


则 $p(\lambda)$ 是关于 $\lambda$ 的 $d$ 次多项式. 引理 3.1 表明

$$
p(\lambda)=\sum_{j=0}^{d}\left(\begin{array}{l}
d \\
j
\end{array}\right) \mathbf{A}_{d+1, k, d-j+1} \lambda^{j}=d !(\lambda+1)^{d} B_{d+1}\left(k+\frac{1}{\lambda+1}\right) .
$$

在方程 $(4.4)$ 的两边关于 $\lambda$ 取 $j$ 阶导数, 则

$$
\begin{aligned}
\mathbf{A}_{d+1, k, d-j+1} & =\left.\sum_{i=0}^{j}(d-j) ! B_{d+1}^{(i)}\left(k+\frac{1}{\lambda+1}\right)\left((\lambda+1)^{d}\right)^{(j-i)}\left((\lambda+1)^{-1}\right)^{(i)}\right|_{\lambda=0} \\
& =d ! \sum_{i=0}^{j}(-1)^{i} \frac{i !(d-j) !}{(d-j+i) !} B_{d+1}^{(i)}(k+1) \\
& =d ! \sum_{i=0}^{j}(-1)^{i} \frac{1}{\left(\begin{array}{c}
d-j+i \\
i
\end{array}\right)} B_{d+1}^{(i)}(k+1) .
\end{aligned}
$$

结合定理 1 和 Hermite 多项式的定义 (4.3), 可以得到

$$
\begin{aligned}
\mathbf{A}_{d+1,\left[x_{d}\right], d-j+1} & =d ! \sqrt{\frac{6}{\pi(d+1)}} \sum_{i=0}^{j}(-1)^{i} \frac{1}{\left(\begin{array}{c}
d-j+i \\
i
\end{array}\right)}\left(\frac{d+1}{12}\right)^{-\frac{i}{2}} D^{i} \exp \left(-\frac{x^{2}}{2}\right)+O\left(d^{\frac{-3}{2}}\right) \\
& =d ! \sqrt{\frac{6}{\pi(d+1)}} \exp \left(-\frac{x^{2}}{2}\right) \sum_{i=0}^{j} \frac{1}{\left(\begin{array}{c}
d-j+i \\
i
\end{array}\right)}\left(\frac{d+1}{12}\right)^{-\frac{i}{2}} H_{i}(x)+O\left(d^{\frac{-3}{2}}\right) .
\end{aligned}
$$

注 4.1 定理 4 表明了细化 Eulerian 数 $\mathbf{A}_{d+1, k, d-j+1}$ 关于充分大的 $d$ 的渐近形式并非 Gauss 函 数. 这一结论解释了 Conger 在文献 [11] 中利用数值实验观测到的现象. 当 $d$ 充分大时, 相邻两细化 Eulerian 数 $\mathbf{A}_{d+1,\left[x_{d}\right], d-j+1}$ 和 $\mathbf{A}_{d+1,\left[x_{d}\right], d-j}$ 之间仅仅相差一个修正项

$$
j !(d-j) ! \sqrt{\frac{6}{\pi(d+1)}} \exp \left(-\frac{x^{2}}{2}\right)\left(\frac{d+1}{12}\right)^{-\frac{j}{2}} H_{j}(x) .
$$

致谢 作者对审稿专家所提出的建议和编辑部的热心帮助表示感谢. 感谢意大利 Lecce 大学初文昌教授和美国 Michigan State University 李天岩教授的讨论和建议.

\section{参考文献}

1 Sommerfeld A. Eine besondere anschauliche Ableitung des Gaussischen Fehlergesetzes. Verlag von J A Barth Leipzig, 1904: 848-859

2 Curry H B, Schoenberg I J. On Pólya frequency functions IV: The fundamental spline functions and their limits. J Anal Math, 1966, 17: 71-107

3 Unser M, Aldroubi A, Eden M. On the asympototic Convergence of B-spline Wavelets to Gabor Functions. IEEE Trans Inform Theo, 1992, 38: 864-872

4 Brinks R. On the convergence of derivatives of B-splines to derivatives of the Gaussian function. Comput Appl Math, 2008, 27: 79-92

5 de Laplace M. Oeuvres Complètes, Vol. 7. Paris: réédité par Gauthier-Villars, 1886

6 Foata D. Distribution Eulérienne et Mahoniennes sur le groupe des permutations. In: Aigner M, ed. Higher Combinatorics. Proceedings of the NATO Advanced Study Institute. Reidel: Dordrecht-Boston, 1976, 27-49

7 Stanley R P. Eulerian partitions of a unit hypercube. In: Aigner M, ed. Higher Combinatorics. Proceedings of the NATO Advanced Study Institute. Reidel: Dordrecht-Boston, 1977, 49 
8 Steingrímsson E. Permutation statistics of indexed permutations. European J Combin, 1994, 15: 187-205

9 Ehrenborg R, Readdy M, Steingrímsson E. Mixed volumes and slices of the cube. J Combin Theory Ser A, 1998, 81: $121-126$

10 Bagno E. Kazhdan constants of some colored permutation groups. J Algebra, 2004, 282: 205-231

11 Conger M. A refinement of the Eulerian numbers and the joint distribution of $\pi(1)$ and des $(\pi)$ in $s_{n}$. Ars Combin, 2010, to appear

12 Wang R H, Xu Y, Xu Z Q. Eulerian numbers: a spline perspective. J Math Anal Appl, 2010, 370: 486-490

13 Tanny S. A probabilistic interpretation of Eulerian numbers. Duke Math J, 1973, 40: 717-722

14 Carlitz L, Kurtz D C, Scoville R, et al. Asymptotic properties of Eulerian numbers. Z Wahrsch Verw Gebiete, 1972, 23: $47-54$

\section{The applications of B-splines in some asymptotic combinatorial problems}

\section{XU Yan \& WANG RenHong}

Abstract In this paper, the convergence order of the derivatives of B-splines is investigated. The asymptotic formulas for Eulerian numbers, refined Eulerian numbers and the coefficients of descent polynomials are obtained directly from the spline interpretations of these numbers. In terms of Hermite polynomials, the asymptotic representations of refined Eulerian numbers are also concluded. The asymptotic formulas for the Eulerian numbers $A_{d, k}$ agree with the previously known results which were given by Carlitz et al., but the convergence order is more accurate. This paper also provides applications of B-splines in the asymptotic combinatorics.

Keywords: B-splines, Eulerian numbers, refined Eulerian numbers, descent polynomials, asymptotic approximation

MSC(2000): 05A16, 41A15 